\title{
The Portuguese air quality management and assessment under INSPIRE and the CAFE Directive
}

\author{
C. Martins \\ Higher Institute of Statistics and Information Management, \\ New University of Lisbon, Portugal
}

\begin{abstract}
The Portuguese Environment Agency (APA) is legally mandated to collect and report to community level the required air quality information in the framework of the existing Air Quality and CAFE Directives.

The Geographic Information National System (SNIG) is the Portuguese Spatial Data Infrastructure (SDI) whose purpose is to provide, from several access points, services for the search, viewing and exploration of geographic information (GI) over Portuguese territory. Other attributions are the coordination of the national GI activities, namely in the INSPIRE context. This will certainly be an essential source of data in order to incorporate data layers of relevant information considered onto concentration mapping and improving the quality of data visualization, which will be incorporated into the GIS. This joint work will be absolutely effective in order to achieve the operability of the actions needed under the INSPIRE requirements.

For the Portuguese Environment Agency (APA) a set of regulated ways for the collection, storage, processing and distribution of main elements concerning the geographical information under the GIS development will be necessary. One of the methods for collecting alphanumeric information after its acquisition would be through a specific database with a convenient data model which could take into account only the relevant information for air quality legislation compliance, namely the new air quality Directive (CAFE) under the INSPIRE system. Hence, GIS plays an important role because of the large amount of geographical data needed in the decision making within the national air quality assessment.
\end{abstract}


This tool helps in the decision support process as a means to obtaining the main information to allow predictions to be made of the possible exceedances to the threshold limits, evaluating the situation and giving recommendations for actions.

Keywords: air quality assessment, INSPIRE, CAFE Directive, GIS.

\section{Introduction}

The EU's Sixth Environment Action Programme (EAP) [1] includes Environment and Health, as one of the four main target areas requiring greater effort and air pollution is one of the issues highlighted in this area. The Sixth EAP aims to achieve levels of air quality that do not result in unacceptable impacts on, and risks to, human health and the environment. A number of problems exist regarding the availability, quality, organisation, accessibility and sharing of spatial information needed in order to achieve the objectives set out in that programme. Development of cost effective and accurate pollution monitoring systems with a mechanism for real time data dissemination of the pollution and air quality index values are essential for successful pollution mitigation measures as well as safe guarding the general public from pending air pollution scenarios (Anjaneyulu [2]).

At a local scale, exposure assessment may be seen as part of a decisionsupport tool for local authorities for improving air quality management. An urban air quality management (UAQM) system should encompass: air quality monitoring, emission inventories, air quality and exposure mapping, air quality and exposure impact assessment of various traffic control strategies in support of the evaluation of action plans, information to the public about past and present air quality levels but also forecasts for next day levels (Jensen et al. [3]).

The environmental data reporting and sharing using the current Internet GIS technology would enable public access to the environmental quality data by interactive map operation, online help and comprehensive background information and it helps to improve their participation in environmental issues and therefore push the government to provide more information and data to the public (Shen [4]).

To enable the Commission to implement efficient data sharing systems over Europe measures that address exchange, sharing, access and use of interoperable spatial data and spatial data services across the various levels of public authority and across different sectors had to be legislated. The INSPIRE Directive establishes the legal requirements for an infrastructure for spatial information in the Community to be established and defines it thus: "Infrastructure for Spatial Information' (SDI) meaning metadata, spatial data sets and spatial data services; network services and technologies; agreements on sharing, access and use; and coordination and monitoring mechanisms, processes and procedures, established, operated or made available in accordance with this Directive" (AGI [5]).

Portugal was one of the first countries in the world with an operational SDI: the SNIG (National Infrastructure for Geographical Information). 
More precisely, the current study puts forward a methodology expected to significantly contribute to implementing provisions of the CAFE Directive to integrate INSPIRE requirements as well as to support a thorough renovation of existing national practices on handling air quality information, including the regional, local level and monitoring networks.

\section{Focus/approach}

The Data Exchange Group (DEG) members have been discussing over the last few years important aspects of the Implementing Provisions (IPs) of the CAFE Directive [6], approved in December 2008 under the Portuguese presidency. Based on the outcomes of these discussions the Group has developed proposals for specific information flows to be included in the IPs and these are presented in the most recent version of DEG's report "Implementing Provisions for Reporting under the Directive on Ambient Air quality and Cleaner Air for Europe", revision of January 2009 [7].

The scope of this study is to address the requirements listed by both the CAFE Directive and draft CAFE Directive IPs and the INSPIRE Directive. It is expected that the future implementation of the CAFE Directive, in line with INSPIRE requirements, will produce an effective European Air Quality Information System, which will enable information on ambient air quality to be available to the Commission, the provision of an efficient exchange information on ambient air quality, an efficient access by the Member States and the public to this information and the compilation of information on ambient air quality on a European scale and support efficient public access.

Addressing these two legislative frameworks, the focus is:

a) the creation of metadata and discovery services making it possible to locate spatial data sets and services on the basis of the content of the corresponding metadata and to display the content of the metadata;

b) transformation services, enabling spatial data sets to be transformed with a view to achieving interoperability;

c) to download services, enabling copies of spatial data sets, or parts of such sets, to be downloaded and, where practicable, accessed directly.

This study will cover the whole chain in Portugal from the point where the information is generated (e.g. air quality monitoring network) and quality controlled and subject to administrative provisions (on a regional, national level) to the intended recipients as specified in the draft CAFE Directive IPs.

\section{Main Portuguese institutions involved}

Several institutions are called to cooperate and synchronize their actions in the INSPIRE context and in the framework of the existing Air Quality and CAFE Directives. 


\subsection{Portuguese legally mandated organizations at national and regional levels}

The Portuguese Environment Agency (APA) (www.apambiente.pt) is acting as a Legally Mandated Organisation (LMO), tasked with the collection and data management, being the national operator and coordinator of the Portuguese Air Quality Network with 77 stations. There are regional LMOs - the Regional Development Coordination Commissions (CCDR) in continental territory and the Environment Regional Directions (DRA) in the national islands who are responsible for collection and data management at regional level, acting as coordinators of observation networks.

\subsection{National geographical institute}

IGP (Portuguese Geographical Institute) is the National Geographical Institute and responsible for the National Geodetic Network and Cadastre.

SNIG is the Portuguese SDI whose purpose is to provide, from several access points, services for the search, viewing and exploration of GI over the Portuguese territory. Other attributions are the coordination of the national GI activities, namely in the INSPIRE context. Also, SNIG was recently updated towards the implementation of the new standards and recommendations from the ISO [8-11] and INSPIRE Directive [12].

\section{Requirements of air quality legislation to take into account in the study}

The analysis of the links and implications of the requirements of the current study identified the pieces of legislation as of relevance for the development of the present work.

The detailed assessment of the CAFE Directive requirements plus associated legislation as identified gives orientation on the following requirements which need to be addressed in detail:

- Various coordinate systems may need to be taken into account when mapping zones and agglomerations in the CAFE/European data model;

- The scale at which assessment methods display air quality assessment over the entire national region will play a crucial role on how air quality results are comparable at the European scale;

- In order to be INSPIRE compliant, there is a need to create a single air quality metadata and data specification profile;

- The usage of commonly available web mapping systems and browsers (e.g. Google Earth and Google Map) to allow quick mapping and visualization of both near real time data (provisional) and ratified data on remote sensing data backgrounds. 


\subsection{On monitoring data services}

An Air Quality Monitoring Facility (AQMF) is composed of the following components as detailed in the data flow presented in Figure 1.

\subsubsection{Data collection}

This component of the AQMF encompasses one or more air quality monitoring station(s) which run specific equipment to register and collect ambient air quality information for legislated pollutants, using a particular method and frequency.

\subsubsection{Data management and validation}

This component deals with data management activities, which include data scaling, polling and calibration. In terms of NRT data sharing purposes (and reporting) as-is or very crudely ratified data needs to be quickly available to the public in an INSPIRE compliant fashion and associated validation issues may play a major challenge to the systems to be devised.

\subsubsection{Data ratification}

The data ratification process plays a major role in the data management flow as it prepares data for both reporting and wider public usage purposes. These data needs to be clearly identified, correction and ratification methods applied clear and associated metadata needs to be explicit and relevant for a successful usage at all levels (reporting, research, policy making, etc.).

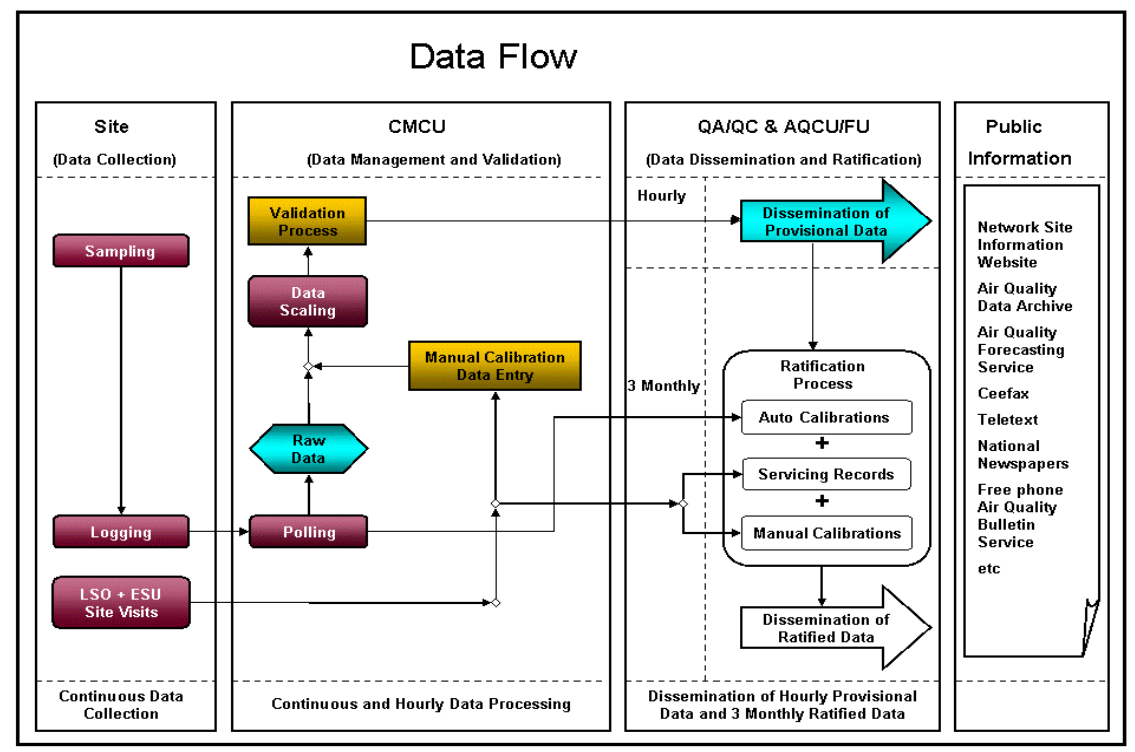

Figure 1: Data flow of an air quality monitoring network facility. 


\subsubsection{Data dissemination}

This data flow stage is the last step in the process of an air quality monitoring facility and links directly with the requirements of both CAFE and INSPIRE Directives. The location, accessibility and usage of appropriate and relevant data by the public is performed during this stage and major challenges include correct metadata definition, database design, INSPIRE compliant metadata and data specifications, projection and coordinate systems, language, clarity of metadata content and web page design and accessibility.

\subsection{On assessment data services}

Assessment methods involve monitoring, expert judgment, modelling or a combination of the two. In order to be able to ultimately produce a reliable and informative SEIS system across Europe to provide relevant and usable air quality assessment information, there is a need to enable concerted methods to map assessment results.

\section{CAFE metadata requirements}

The methodology used to define the common metadata profile pursues the following steps:

- Identification of associated user requirements focusing on CAFE reporting and exchange of information requirements (EoI) whilst simultaneously accounting for public information and wider dissemination.

- CAFE specific user requirements were extracted from the following sources:

i. Data Exchange Module - DEM requirements;

ii. Questionnaire form requirements (annual report on air quality assessment and management (2004/461/EC [13] obligations) and

iii. DEG document as of January 2009 specifications. This includes the Exchange of Information (EoI Data on air quality, Council Decision 97/101/EC [14]) requirements, which were discussed and defined by the group to a good level of detail and specified in the Group's report [7].

Once all the requirements were identified, that content must be mapped against Commission Regulation (EC) No 1205/2008 [15] to extract the INSPIRE related metadata elements for the CAFE/INSPIRE metadata profile definition.

\subsection{Identification of main data classes of CAFE user requirements}

Directive 2008/50/EC reporting obligations and data exchange between Member States are currently fulfilled via four main mechanisms, still in compliance with the repealed Air Quality Framework Directive (96/62/EC) as follows:

i. Questionnaire - The EU air quality legislation requires the Member States to report on zones designated under the CAFE IP for each pollutant and to report annually on the levels in comparison with air quality objectives (reporting); 
ii. Data Exchange Module (DEM) as at http://airclimate.eionet.europa.eu/country_tools/aq/aq-dem/(data exchange);

iii. On-line data and alert messages to the public when Limit Value is exceeded (reporting and exchange of information).

Having taken into account the reporting and data exchange mechanisms listed above and the DEG document requirements (as of 23rd January 2009) [7], the following main classes of user requirements were identified:

i. LMOs/responsible entities;

ii. Monitoring facility datasets (include network, station and measurement configuration information);

iii. Monitoring data or observation datasets (measurements);

iv. Assessment datasets (compliance/exceedence);

v. CAFE zones (agglomerations and non-agglomeration zones).

\subsubsection{Entities (LMOs and other responsible organizations)}

The Entities dataset describes the various organisations with responsibility for air quality data (data collection, validity and quality assurance - management and QA/QC procedures - assessment, reporting, ownership and wider dissemination).

\subsubsection{Monitoring facilities}

The Monitoring Facilities dataset describes the monitoring network/stations used for reporting, data sharing and compliance progress checking purposes. The Monitoring Facility dataset can be used to describe an individual environmental monitoring facility or a monitoring network.

\subsubsection{Observation datasets}

The Observation dataset is an abstract dataset that can be used to describe the results of observed properties acquired using an appropriate procedure or method.

\subsubsection{Assessments datasets}

The Assessment dataset is an abstract dataset that can be used to describe the methods and results of derived observations, modelled results, expert estimation or a combination of methods and is in line with the dataflows agreed by the Data Exchange Group.

\subsubsection{Zones and agglomerations}

The Zones and Agglomerations dataset provides descriptive information that can be used to discover as well as to see trends in both the spatial extent of zones as pollutant levels change, and trends in pollutant levels zone(s) with time.

\section{INSPIRE metadata requirements}

As stated in Commission Regulation (EC) No 1205/2008 [15], this is the minimum set of metadata elements necessary to comply with Directive 
2007/2/EC and does not preclude the possibility for organisations to document the information resources more extensively.

\subsection{Legal provisions}

The INSPIRE Directive requires metadata to be provided on the following types of information:

a) conformity of spatial data sets with the implementing rules;

b) conditions applying to access to, and use of, spatial data sets and services and, where applicable, corresponding fees;

c) quality and validity of spatial data sets;

d) public authorities responsible for the establishment, management, maintenance and distribution of spatial data sets and services;

e) limitations on public access and the reasons for such limitations.

\subsection{Technical requirements under INSPIRE services}

INSPIRE foresees the establishment of more detailed provisions that are called Implementing Rules need to be established for:

\subsubsection{Metadata}

The main purpose of metadata is to document information resources and facilitate their re-use by the resource owner, and others either within or out of the original community of interest. INSPIRE metadata shall include information on the conformity of spatial data sets with the implementing rules, the rights of use of spatial data sets and services and as well the quality and validity of spatial data (Tóth [16]).

\subsubsection{Interoperability of spatial data sets and services}

The implementing rules provided for in INSPIRE Directive shall be designed to ensure that it is possible for spatial data sets to be combined, or for services to interact, in such a way that the result is a coherent combination of spatial data sets or services that represents added value, without requiring specific efforts on the part of a human operator or a machine (Tóth [16]).

\subsubsection{Network services (geo-portal)}

Network services are necessary for sharing spatial data between the various tiers of public authorities in the community (Tóth [16]). In order to assist the integration of the national infrastructures into the infrastructure for spatial information in the community, Portugal should provide access to your infrastructure through a community geo-portal operated by the Commission, as well as through any access points that decides to operate.

\subsubsection{Policy for data and service sharing}

Portugal shall adopt measures for the sharing of data and services between public authorities for public tasks relating to the environment without restrictions occurring at the point of use. 
When spatial data or services are provided to community institutions for reporting obligations under community law relating to the environment then this will not be subject to charging.

\subsubsection{Coordination and measures for monitoring reporting}

An efficient environmental monitoring data sharing is extremely important being an invaluable tool to support environmental studies and strategies as well as enabling informed environmental policy decisions. The problems regarding the availability, quality, organization, accessibility and sharing of spatial information are common to a large number of policy and information themes. Such constrains are largely experienced across the various levels of public authority and need to be identified and solved for a successful European wide data sharing system (Grossinho and Wilson [17]).

\section{Outlook for the future and streamlining air quality issues}

In the context of the CAFE Directive agreed during the second reading procedure in December 2008 under the Portuguese presidency, the data exchange and reporting procedures are expected to differ significantly from the current legislative requirements and associated defined formats.

Currently, each member state collects and publishes their air quality monitoring and assessment data using different systems and designs and their methods for reporting and data publishing strategies reflect their national mechanisms for air quality estimation and data sharing decisions.

\subsection{Potentialities of use INSPIRE services on air quality management and assessment under CAFE Requirements - benefits of using}

The application of GIS can bring clear advantages for air quality management activities, related to the general benefits of improved efficiency and flexibility of data handling and representation.

These include the ability to integrate different spatial and attribute data sets such as pollutant source data with activity and the use of a variety of spatial analysis techniques to help with assessing compliance with air quality objectives.

GIS techniques are capable of providing geospatial air quality models, i.e., at any time and any location anyone can access the Air Quality Status (AQS) of that area (Jensen [18]).

A well-defined Decision Support System (DSS) under GIS environment may be developed so that Air Quality Monitoring System (AQMS) may work efficiently. This will help in taking the decisions to improve the present air quality status by means of making rules and regulations by the concerned authorities (Dalh [19]).

The INSPIRE implementation will follow a step-wise approach, starting with unlocking the potential of existing spatial data and spatial data infrastructures and then gradually harmonising data and information services allowing 
eventually the seamless integration of systems and datasets at different levels into a coherent European spatial data infrastructure (RDM Working Group [20]).

It is recognized that the more the software industry (for instance, relating to GIS software) supports these standards in its products, the easier it will be for those responsible for the implementation of INSPIRE to make systems in the Member States comply with the standards, thus improving the cost-effectiveness of the implementation of the Directive (Annoni and Craglia [21]).

Under the current air quality reporting and data sharing legislative requirements (2004/461/EC [13]) data are currently provided through a variety of methods and formats which do not easily allow data to be interoperable across other environmental thematic areas. It is clear that the importance of developing a Portuguese strategy to successfully reach the implementation of the CAFE Directive under INSPIRE services in order to get, in particular, support of spatial representation of assessment as well technical solutions for semi-automated aggregation of data sets and strong support for the automation of quality control and verification tools. Also, seamless access to environmental information through the standardisation of services and underlying specifications for the discovery, view, access, download, transformation and invocation of spatial data sets. These several tasks are managed by many different public authorities, on different levels of the administrative hierarchy and within different administrative units.

Hence, for the spatial datasets relating to Annex III of the INSPIRE Directive and the reporting requirements of the CAFE Directive, there are a few challenges that need to be addressed across various Member states in order to fulfil both INSPIRE and CAFE requirements. Under these, some critical components have been identified:

- the level of knowledge and understanding of the INSPIRE Directive requirements at the various LMO levels;

- the level of knowledge and understanding of EMF data usage for reporting purposes under current and future EC legislation by geographic information systems (GIS) professionals;

- communication issues at the technical level; the need to speak the same technical language or at least to make sure communication is effective. Air Quality specialists do not follow the technical terms required for INSPIRE implementation.

\section{Conclusion}

To manage the environment effectively the EU needs the better provision of spatial data provision to support environmental policy making, better spatial data flows between systems to support these policies and better sharing of this data between governments, agencies and the citizen. A lack of standards for the provision of spatial data and no pan-EU directives for sharing or coordinating the use of this, have driven the need for INSPIRE.

The assessment of several NSDI in Europe shows that all the countries have basic components of a national SDI, which provide a sound basis for the 
development of the European SDI, i.e. INSPIRE. The assessment also shows that NSDI development is not equal throughout Europe. All countries will need to analyse their current status in more detail and compare it with the requirements of INSPIRE as defined through the Implementing Rules for metadata, data interoperability, network services, data sharing, and monitoring and reporting.

In Portugal a set of regulated ways for the collection, storage, processing and distribution of main elements concerning the geographical information under the GIS development will be needed. One of the methods for collecting alphanumeric information after its acquisition would be through a specific database with a convenient data model which could take into account only the relevant information for air quality legislation compliance, namely the new air quality Directive (CAFE) under the INSPIRE system.

It is extremely important that the anticipation about both knowledge of the INSPIRE requirements and the way which the new CAFE Directive makes its influence on the air quality data and metadata harmonization and interoperability through the MS and in particular within Portugal. Taking this into account the exercise performed has been useful regarding the preparation and awareness of technicians belonging to the different LMOs and better understanding about the future implications of the way both Directives (INSPIRE and CAFE) must synchronize.

\section{References}

[1] COM 46 final, Brussels. Towards a Shared Environmental Information System (SEIS), 2008.

[2] Anjaneyulu, Y., Continuous air pollution monitoring and GIS for episode warning in urban environment, Environmental Science and Engineering, May India, Vol. 2., 2004.

[3] Jensen SS, Berkowicz R, Hansen HS, Hertel O., A Danish decision-support GIS tool for management of urban air quality, Transp Res., Part D 6(4): $229-41,2001$.

[4] Shen, L., Internet GIS for Air Quality Information Service for Dalian, China, Master of Environmental Studies en Geography, University of Waterloo, Ontario, Canada, 2005.

[5] AGI, The INSPIRE Directive - A brief Overview, Association for Geographic Information, 2007.

[6] COM, Directive on ambient air quality and cleaner air for Europe (CAFE) 2008/50/EC, 2008.

[7] Draft - DEG Working Material - Implementing Provisions for Reporting under the Directive on Ambient Air quality and cleaner air for Europe, 2009.

[8] EN ISO 19115:2003, Geographic information-Metadata, 2003.

[9] EN ISO 19115:2003/Cor 1:2006, Geographic information - Metadata, 2006.

[10] ISO 19119:2005, Geographic Information-Services, 2005. 
[11] ISO 19119:2005/Amd 1:2008, Extensions of the service metadata model, 2008.

[12] Directive 2007/2/EC of the European Parliament and of the Council of 14 March 2007, Establishing an Infrastructure for Spatial Information in the European Community (INSPIRE), 2007.

[13] COM, Commission Decision laying down a questionnaire to be used for annual reporting on ambient air quality assessment, 2004/461/EC, 2004.

[14] COM, EoI Data on air quality, Council Decision, 97/101/EC, 1997.

[15] Commission Regulation (EC) No 1205/2008 Implementing Directive 2007/2/EC of the European Parliament and of the Council as regards metadata, 2008.

[16] Tóth, K., INSPIRE - First steps towards the Environmental Spatial Data Infrastructure in Europe from point of view of cartography, International Conference on cartography and GIS, Borovets - Bulgaria, 2006.

[17] Grossinho, A. and Wilson, D., Prototype of INSPIRE technical specifications for air, water, waste and biodiversity environmental monitoring facilities, INSPIRE Conference, Maribor - Slovenia, 2008.

[18] Jensen, S.S., A Geographic Approach to Modelling Human Exposure to Traffic Air Pollution using GIS, Ph.D. thesis, National Environmental Research Institute, Denmark, 1999.

[19] Dalh, I.M., GIS Based Environmental Decision support System, Asian Conference on Remote Sensing, 1997.

[20] RDM Working Group, Reference Data and Metadata, Position Paper. INSPIRE RDM PP v4-3 en., 2002.

[21] Annoni, A. and Craglia, M., Towards a Directive Establishing an Infrastructure for Spatial Information in Europe (INSPIRE), From Pharaohs to Geoinformatics. Cairo, Egypt April 16-21, 2005. 